\title{
Effect of a multi-ingredient based food supplement on sexual function in women with low sexual desire
}

\author{
S. Palacios ${ }^{1 *} \mathbb{D}$, E. Soler ${ }^{1}$, M. Ramírez ${ }^{1}$, M. Lilue ${ }^{1}$ D. Khorsandi ${ }^{2,3}$ and F. Losa ${ }^{4}$
}

\begin{abstract}
Background: Studies have demonstrated that women with low desire and low excitement have negative feelings regarding their physical and emotional satisfaction, as well as their happiness. In this study, we evaluate the efficacy of Libicare ${ }^{\varpi}$ - a multi-ingredient food supplement - to improve sexual function in postmenopausal women.

Methods: This was an exploratory, prospective, non-controlled, observational study. Postmenopausal women aged 45-65 with a risk of sexual dysfunction (Female Sexual Function Index (FSFI) $<25.83$ ) were included during routine clinical visits and treated with 2 tablets of Libicare ${ }^{\circledast}$ daily for 2 months. Libicare ${ }^{\circledast}$ is an oral food supplement containing Trigonella foenum graecum, Turnera diffusa, Tribulus terrestris, and Ginkgo biloba dry extracts. Primary endpoint: change vs. baseline in FSFI score. Secondary endpoints: 1) changes in testosterone and serum steroid levels of free testosterone and sex hormone-binding globulin (SHBG) levels and 2) tolerability.
\end{abstract}

Results: A total of 29 patients (mean age: 54.69 years) were included. FSFI mean (SD) score showed a significant increase: 20.15 (4.48) vs 25.03 (6.94), baseline vs final; $p=0.0011$, paired t-test. Most patients (86.2\%) increased their FSFI score. All FSFI domains, except dyspareunia, showed significant increases. The highest increase was observed in the desire domain $(p=0.0004)$. Testosterone and SHBG levels were assessed in 21 patients. A significant increase in testosterone level was observed: 0.41 (0.26) vs. 0.50 (0.34) pg/mL, baseline vs. final; $p=0.038$, Wilcoxon test. $52.4 \%$ of patients increased their testosterone levels. Finally, a significant decrease was observed in SHBG level: 85 (32.9) vs. 73 (26.8) nmol/L, baseline vs. final; $p=0.0001$; paired t-test. $95.2 \%$ of patients decreased their SHBG levels.

Conclusion: In this pilot study, a significant improvement in sexual function and related hormone levels was observed with Libicare ${ }^{\oplus}$. Further studies must be conducted to confirm these exciting results.

Trial registration: Current Controlled Trial ISRCTN12928573. Date of registration: 28/March/2019. Retrospectively registered.

Keywords: Sexual function, Low sexual desire, Trigonella foenum graecum, Testosterone

\section{Background}

Low sexual desire affects more than $20 \%$ of women [1]. A survey among American and European women (Women's International Study of Health and Sexuality, WISHeS) evaluated the prevalence of hypoactive sexual desire $[2,3]$. Among European women, aged 20-49, in their reproductive age, the prevalence of reduced desire causing discomfort and concern was $7 \%$. In women aged $50-70$, the prevalence was $9 \%$ in women with natural

\footnotetext{
* Correspondence: ipalacios@institutopalacios.com

'Gynaecology and Obstetrics Department, Palacios' Institute of Women's Health, C/Antonio Acuña 9, E-28009 Madrid, Spain

Full list of author information is available at the end of the article
}

menopause, and $12 \%$ in women subjected to surgical menopause [2].

After interviewing 750 women, the most common sexual issues described in the Spanish population were lack of sexual interest (36.0\%), impossibility of having an orgasm (27.8\%), and unsatisfactory sexual relationships (25.1\%) [4].

Women with low sexual desire may have problems when starting or having stable sexual relationships, and they may feel unsatisfied and experience marital disorders. Studies have demonstrated that women with low desire, low excitement, or sexual pain are clearly associated with negative feelings regarding their physical and

(c) The Author(s). 2019 Open Access This article is distributed under the terms of the Creative Commons Attribution 4.0 International License (http://creativecommons.org/licenses/by/4.0/), which permits unrestricted use, distribution, and 
emotional satisfaction, as well as their happiness. In addition, women suffering from those problems tend to experience much more negative emotions and psychological states than women with normal sexual activity $[5,6]$.

The 5th edition of the Diagnostic Statistical Manual of Mental Disorders, (DSM-V, 2013) [7] features some changes as an attempt to correct, extend, and clarify the different diagnoses related to sexual dysfunction and their criteria. For instance, they have brought female desire and excitement disorders together under a single diagnosis called 'Female Sexual Interest/Arousal Disorder'.

Biological, psychological, and interpersonal factors play a part in sexual dysfunction. The hormones impacting female sexuality (estrogens and androgens) play a crucial role in sexual function [8]. Various studies have demonstrated that reduced testosterone levels are directly related to decreased sexual activity in postmenopausal women $[9,10]$. However, the fact that women produce higher amounts of androgens than estrogens demonstrates how important their role is. Having a deeper knowledge of intracrinology may potentially facilitate a better understanding of the correlation between androgen levels and sexual desire [11].

There is clear clinical evidence that strongly suggests that androgens play a vital role in sexual dysfunction, taking into account the findings published on Trigonella regarding increased testosterone blood levels and improved sexual function and desire [12-14]; as well as the potentially beneficial effects on sexual function published on Turnera diffusa as a regulator of the natural balance between androgens and estrogens [15-17]; in addition to Tribulus terrestris [18] and Ginkgo biloba [15-17] to facilitate blood flow and to provide a relaxing effect on smooth muscle. Overall, studying the effect of an extract with all these components on women with low sexual desire was deemed necessary. We presented this data at the 21st World Meeting on Sexual Medicine as a poster [https:// www.sciencedirect.com/science/article/pii/S174360951830 4399?via\%3Dihub].

Therefore, the objective of this pilot study is to evaluate the efficacy of a multi-ingredient food supplement, marketed under the trade name Libicare ${ }^{\oplus}$, to improve sexual function in postmenopausal women.

\section{Methods}

This is an observational, prospective, non-controlled pilot study scheduled for 30 postmenopausal participants aged 45-65 with a Female Sexual Function Index (FSFI) $<25.83$, this number being considered as the threshold for low sexual dysfunction risk [19]. All women were included at routine clinical visits and

were treated with 2 tablets of Libicare ${ }^{\oplus}$, one every $12 \mathrm{~h}$, daily for 2 months ( 9 weeks). Libicare ${ }^{\oplus}$ is an oral food supplement containing dry extracts of Trigonella foenum graecum, Turnera diffusa, Tribulus terrestris, and Ginkgo biloba (see Table 1). Libicare ${ }^{\curvearrowleft}$ is manufactured by Procare Health (Barcelona, Spain). The primary endpoint was to evaluate the efficacy on sexual function. Risk of sexual dysfunction was evaluated using the FSFI. The FSFI is a 19-item questionnaire divided into six domains: desire, arousal, lubrication, orgasm, satisfaction, and pain [20-23]. Scoring system: the individual score was achieved and then added to other scores from the same domain, multiplied by the relevant factor. The total scale was achieved by adding the scores obtained in the 6 domains. This tool provides optimal psychometric properties for each of the 6 domains, is easy to use, and has been demonstrated to be able to discriminate between clinical populations (women with risk of sexual dysfunction) and non-clinical populations (without sexual disorders).

A selection period during which other inclusion criteria apart from the FSFI index were also evaluated was followed by a 9 -week treatment period.

\section{Inclusion criteria}

- Healthy, postmenopausal women (no natural menses for at least 1 year) aged $\geq 45$ and $\leq 65$. Hysterectomized patients should have an FSH level above $40 \mathrm{IU}$.

- Women with a stable partner, living together for at least 15 days a month and being sexually available.

- Risk of sexual dysfunction established at FSFI score $<25.83$.

- Integrity of the vaginal mucosa (without lesions or bleeding).

- Women willing to and capable of understanding and signing an informed consent after receiving an explanation on the nature of the whole study.

- Consenting to participate in the study and signing the Informed Consent form.

- No desire for pregnancy in the next 3 months.

Table 1 Libicare ${ }^{\oplus}$ nutritional information

\begin{tabular}{lll}
\hline Nutritional Information & For 2 tablets & $\begin{array}{l}\text { Nutrient Reference } \\
\text { Value (NRV) }\end{array}$ \\
\hline Dried extract of Trigonella & $600 \mathrm{mg}$ & - \\
- Saponines & $300 \mathrm{mg}$ & - \\
Dried extract of Damiana & $150 \mathrm{mg}$ & - \\
Dried extract of Ginkgo & $50 \mathrm{mg}$ & - \\
- Ginkgo flavonoids & $12.50 \mathrm{mg}$ & - \\
Dried extract of Tribulus & $40 \mathrm{mg}$ & - \\
- Saponins & $36 \mathrm{mg}$ & - \\
Vitamin B3 & $32 \mathrm{mg}$ & 200 \\
Vitamin B2 & $2.8 \mathrm{mg}$ & 200 \\
Vitamin B1 & $2.2 \mathrm{mg}$ & 200 \\
Vitamin B6 & $2 \mathrm{mg}$ & 143 \\
Selenio & $50 \mathrm{mg}$ & 90.91 \\
\hline
\end{tabular}




\section{Exclusion criteria}

- Non-compliance with the requirements above.

- Pregnant women or with suspected pregnancy.

- Within 3 months following delivery or abortion.

- Breastfeeding women.

- Women with severe pain in sexual relationships (DMS-V).

- Non-diagnosed abnormal genital bleeding or presence of vaginal lesions.

- Women with symptoms of vaginal infection or signs of any other genital infection.

- Women allergic or hypersensitive to the components of the study treatment.

- Severe psychiatric disorder.

- Use of any hormonal treatment with estrogens, progestogens, or estrogens and progestogens within 3 previous months prior to selection.

- Use of any other drug or experimental device within 30 days prior to selection.

- Any condition preventing the patient from participating in the study, at the researcher's discretion.

All participants received a written consent form and signed it before any procedure related to the study was carried out. Participants underwent a medical history review, medical examination, and comprehensive gynecological check-up. A gynecological examination was performed to assess the appearance of the mucosa and the tolerance to medication on day 1 and on week 9 .

\section{Laboratory tests}

Serum steroid levels of free testosterone and sex hormone-binding globulin (SHBG) were measured in the LABCO Laboratory. SHBG was measured with chemiluminescent immunoassay Immulite $2000 \mathrm{XPi}$ (Siemens Healthcare Diagnostics, Eschborn, Germany) with an inter-assay coefficient of variation of 3.5 and $8.3 \%$ at the low level, and 4.8 and $5.4 \%$ at the high level, respectively. The estimation of serum free testosterone hormone levels was carried out using the ELISA technique. The DiaMetra Italy kit (DKO-015) was used to determine free testosterone hormone concentration in human serum according to the manufacturer's instructions.

FSFI was performed in the whole sample (29 individuals), and determinations of free testosterone and SHBG were achieved in 21 individuals [20]. Hormonal determination was only performed in patients giving their consent to that. The characteristics of the analytical parameters (FSFI free testosterone and SHBG) in the two visits (pre/post), the absolute change between visits (difference post-pre), and the relative change between visits ([post-pre]/pre $\times 100 \%$ ) were described.

\section{Statistical methodology}

A descriptive analysis of the variables included in the study was carried out. Central tendency and dispersion measures were presented, that is, the mean, standard deviation (SD), median, 25th and 75th percentiles (P25 and P75, respectively), and minimum and maximum (min. and max., respectively) of quantitative variables.

Absolute (n) and relative (\%) frequency distributions of qualitative variables were presented. Unavailable data were not allocated. They were just described as lost data.

To analyze the evolution/change throughout the study, parametric tests for continuous variables (Student's $T$ test for paired samples) and/or non-parametric tests (Wilcoxon) were used.

The hypothesis testing was two-tailed in all cases, with a significance level of $p<0.05$. Analyses were carried out using the SAS statistical software, version 9.4.

\section{Results}

The sample to be analyzed was made up of 29 patients who completed the study. One patient withdrew from the study as she had to travel. Of the 29 patients, 21 consented to blood tests for hormonal determination prior to and upon completion of the study.

Demographic data from patients are shown in Table 2. Mean age was 54.69 (45-65). All women were Caucasian and from varied educational and economic backgrounds. In this study group, all women were considered as healthy based upon clinical history, examination, and recent blood and biochemistry tests less than 3 months old.

FSFI results are shown in Table 3 and Fig. 1, which features the mean of baseline values and values after two months, with their standard deviations, for each domain, as well as the global determination. As demonstrated by the results after treatment, values were significant for all

Table 2 Demographic characteristics $(n=29)$

\begin{tabular}{ll}
\hline Age (years, mean SD) & $54.69(6.29)$ \\
Weight (Kg, mean SD) & $63.33(8.81)$ \\
Height (cm, mean SD) & $159.40(7.06)$ \\
Academic level (n, \%) & \\
Primary & $11(39.2 \%)$ \\
Secondary & $8(28.5 \%)$ \\
Graduated & $9(32.3 \%)$ \\
N/A & 1 \\
Gynecological characteristics & \\
Mean menarche age (SD) & $12.62(1.5)$ \\
Mean menopause age (SD) & $49.42(6.92)$ \\
Hysterectomy n\% & $4(13.8 \%)$ \\
Mean pregnancies & $1.23(1.06)$ \\
Mean deliveries (SD) & $1.03(1.09)$ \\
\hline
\end{tabular}


Table 3 Mean of baseline values and values after two months of Libicare ${ }^{\circledast}$ treatment. Absolute change and statistical significance of the different FSFI domains

\begin{tabular}{|c|c|c|c|c|}
\hline & Mean at baseline & Mean at 2 months & Absolute change (mean) & SS \\
\hline Desire & $2.40(0.96)$ & $3.33(1.29)$ & $0.93(1.25)$ & $p=0.0004$ \\
\hline Excitement & $3.04(0.96)$ & $3.63(1.44)$ & $0.59(1.25)$ & $p=0.0166$ \\
\hline Lubrication & $3.70(0.98)$ & $4.50(1.34)$ & $0.80(1.34)$ & $p=0.0034$ \\
\hline Orgasm & $3.57(1.14)$ & $4.23(1.43)$ & $0.66(1.30)$ & $p=0.0106$ \\
\hline Sexual satisfaction & $4.01(0.99)$ & $4.62(1.22)$ & $0.61(1.45)$ & $p=0.0321$ \\
\hline Dyspareunia & $4.19(1.06)$ & $4.76(1.40)$ & $0.57(1.55)$ & $p=0.0602$ \\
\hline Global & $20.42(4.23)$ & $25.08(6.62)$ & $4.15(6.14)$ & $p=0.0011$ \\
\hline
\end{tabular}

domains except for dyspareunia. The domain presenting the highest increase was desire, with $p=0.0004$. The global domain increased 4.15 (6.14) points on average, with a statistical significance of $p=0.0011$.

There were 18 patients, accounting for $62 \%$ of subjects, who initially had sexual dysfunction risk scores and eventually reached normality, with their FSFI increasing beyond 25.83. A total of $86.2 \%$ of patients experienced an increase in their FSFI following treatment (Fig. 1).

As far as free testosterone data are concerned, Fig. 2 shows the numbers at visit 1 (pre) and visit 2 (post), as well as the change between both visits. With a sample of 21 patients with available data, statistically significant differences were noted in testosterone values between visit 1 (pre) and visit 2 (post). Testosterone mean change between visits (post-pre) was an increase of 0.09 (SD $0.17)$ units $(p=0.0386)$; the mean percent change as compared to baseline was $79.3 \%(p=0.0214)$.

Finally, SHBG data at visit 1 (pre) and visit 2 (post), as well as changes between both visits, are shown in Fig. 3. With a sample of 21 patients with available data, statistically

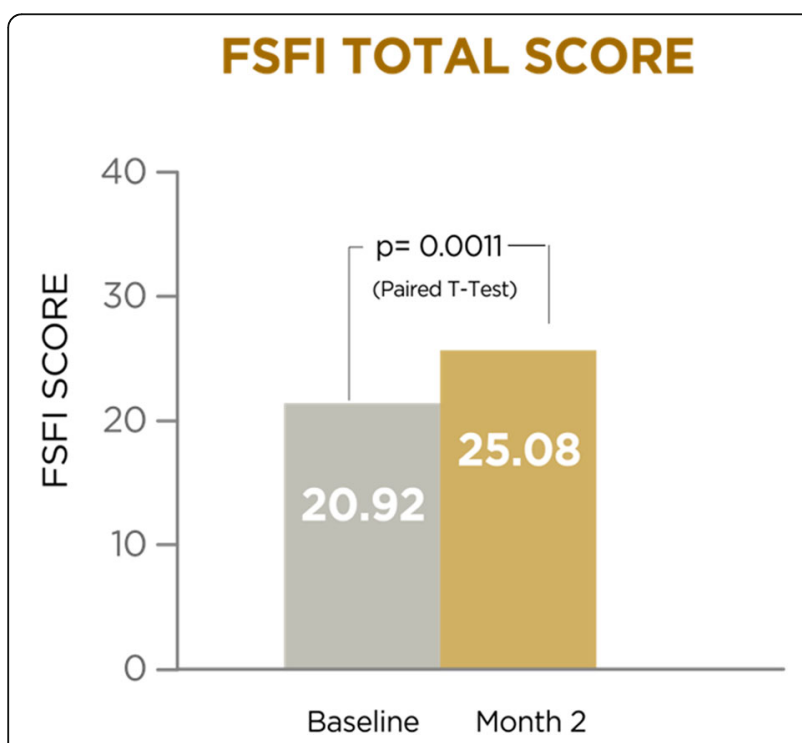

Fig. $1 \mathrm{FSFI}$ total score at baseline and after 2 months of Libicare significant differences were noted in SHBG values between visit 1 (pre) and visit 2 (post). The mean SHBG change between visits (post-pre) represented a decrease of -12.05 (SD 11.48) units $(p=0.0001)$; the mean percent change as compared to baseline was $-13.17 \%$ ( $p<0.0001)$. $95.2 \%$ of patients assessed experienced a decrease in SHBG values (only one patient had increased SHBG values).

\section{Discussion}

The hormones that influence female sexuality are estrogens and androgens. Several studies have shown that a reduction in testosterone level is directly related to a decrease in sexual activity in postmenopausal women $[9,10]$. However, it is also true that other studies have shown that the level of testosterone is not related to sexual performance [24, 25]. On the other hand, there is increasing data pointing to a possible role of androgens on the neurotransmitter dopamine and serotonin [26, 27]. Dopamine plays an essential role in the modulation of sexual desire; it seems to increase sexual desire, the subjective sensation of excitement and the desire to continue sexual activity once the stimulation has begun $[27,28]$.

Although the fact of testosterone's effectiveness as a treatment in female sexual interest disorders has already been demonstrated, there are some doubts regarding its long-term safety [29-32]. This is the reason that the US Food and Drug Administration avoided authorizing testosterone patches to be marketed [33] and paving the way for new strategies to fight this disorder.

One of them is flibanserin, a $5 \mathrm{HT}_{\mathrm{a}}$ agonist and a $5 \mathrm{HT} 2$ a antagonist, which has been demonstrated to increase sexual desire, reduce sexual dysfunction-associated discomfort, and improve sexual activity in premenopausal women with low sexual desire, with a good tolerability [34, 35], which translated into flibanserin being approved in the US [36]. However, this central nervous system drug also has some side effects, which has led to some controversy [37], naturally paving the way for new products to treat and improve low female sexual interest.

Therefore, investigating new therapeutic options based on safe natural products which have been widely used 


\section{SHBG}
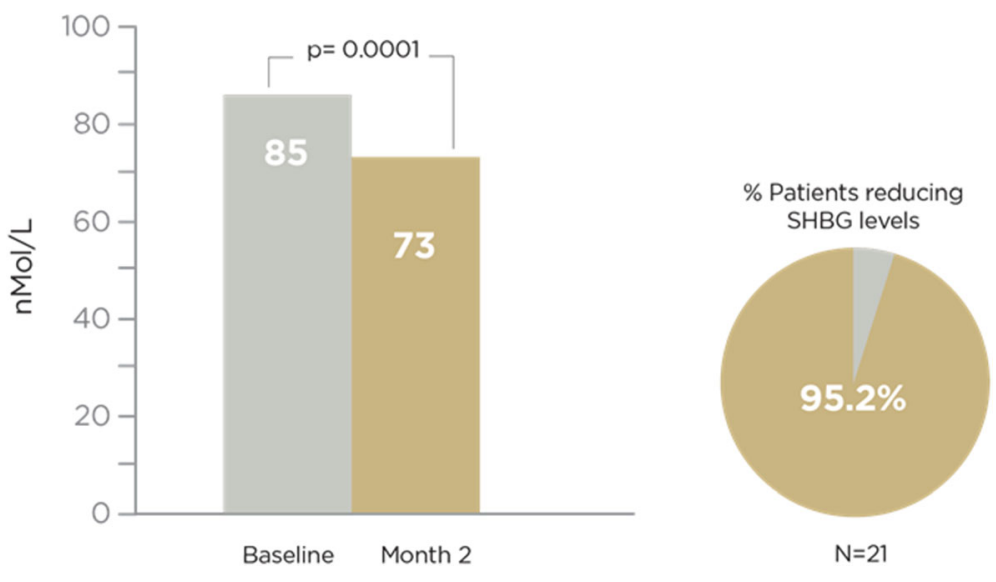

Fig. 2 Free testosterone at baseline and after 2 months of Libicare

for decades and have been demonstrated as effective for sexual desire and arousal seems more than reasonable.

The results from this study, using a multi ingredientbased product including Trigonella foenum graecum, Turnera diffusa, Tribulus terrestris, and Ginkgo biloba, made us think it may have a potential double effect, improving sexual desire through an increase in free testosterone, but also improving arousal by means of a vaginal vasodilating effect. All these effects with the separate components are widely described in numerous studies [12-18], https://www.sciencedirect.com/science/article/pii/S1743609 518304399?via\%3Dihub.

In our study, the whole array of natural components Libicare $^{\bullet}$ consists of has demonstrated a statistically significant improvement in desire, arousal, lubrication, orgasm, and sexual satisfaction domains. Indeed, the global analysis of all FSFI domains demonstrated a significant improvement of more than 4 points after 2 months of treatment,

Dyspareunia was the only domain showing improvement without statistical significance. This was to be expected as one of the exclusion criteria in this study was severe pain in sexual relationships. Owing to its characteristics, this product can be used to address female desire and arousal disorders, that is, the Female Sexual Interest/Arousal Disorder described in the DSM-V [7]. In addition, dyspareunia domain data are close to significance after 2 months of treatment. If used longer, the improvement could possibly achieve statistical significance.

\section{TESTOSTERONA}
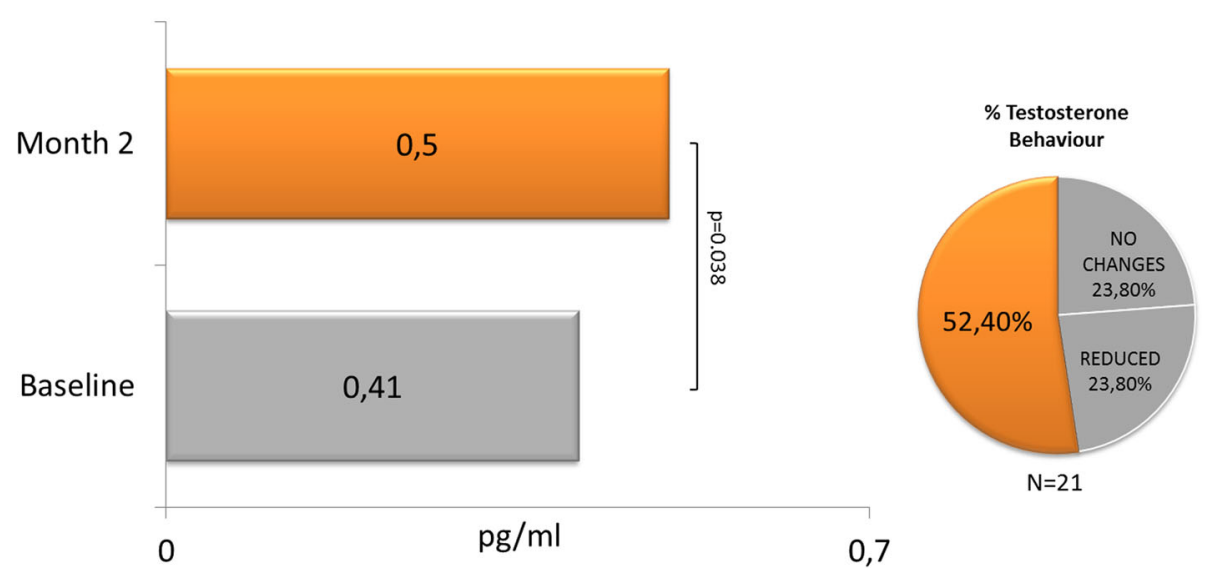

Fig. 3 SHBG at baseline and after 2 months of Libicare 
Most patients improved their FSFI score following 2 months of Libicare ${ }^{\circ}$ treatment with $62 \%$ of them achieving normality rates. Only $13.8 \%$ of women had no improvement whatsoever or a decrease in FSFI score. Even though this is a small percentage, this possibly demonstrates the relevance of the multifactorial causes of this disorder and how important treatment selection and individualization is.

As we already said, this improvement with the use of Trigonella foenum graecum, which proves especially significant regarding sexual desire, had already been found by other authors. Indeed, Amanda Rao et al. [14] demonstrated that a specialized extract of Trigonella foenum graecum seed has a positive impact on the improvement of sexual function, specifically in both sexual desire and arousal, in healthy menstruating women with low sexual function. In our case, patients were postmenopausal and older (45-65), with equally positive results. Furthermore, Elham Aktari et al. [38], used Tribulus terrestris in women with hypoactive sexual desire, and after 4 weeks, they found statistically positive effects on the FSFI score.

The significant increase observed in free testosterone consolidates the results and explains the rise of sexual desire. It is worth noting that this increase is modest and within normal levels, and it is also correlated with the significant decrease found in SHBG levels. Both free testosterone and SHBG hormonal changes have already been demonstrated with Trigonella foenum-graecum extracts by other authors [14].

This pilot study has two limitations: a small number of women included and no control or placebo group. Therefore, these clinical results should be reinforced with other randomized, placebo-controlled studies. On the other hand, the small size of the sample does not allow knowing the importance of these results.

The administration of this product is associated with a significant increase not only in desire and arousal, but also in vaginal lubrication and orgasm. As previously explained, this occurs as part of a domino effect which makes other sexual domains improve when one of them does, and also as a double effect of this product on increasing free testosterone and local vasodilatation. The effect and activity of Turnera diffusa and Ginkgo biloba, the first with an anti-aromatase activity [39], and the second with a vasodilation activity [40], account for the improvement in these two domains (lubrication and orgasm).

There are very few studies analyzing the effect of Trigonella foenum-graecum on female sexual function. The results from our work support others previously found [14]. Additionally, the possibilities of the various components of this product pave the way to understand the effects on the different domains of sexuality and the double effect, both on desire and arousal.

\section{Conclusion}

In this pilot study, the multi-ingredient food supplement, Libicare ${ }^{\bullet}$, has shown an improvement in desire, arousal, lubrication, orgasm, and sexual satisfaction domains, with a clear increase in free testosterone numbers and a decrease in SHBG levels in postmenopausal women. It is necessary to perform a randomized study with a control group to confirm these results.

\section{Abbreviations \\ DKO-015: DiaMetra Italy kit; DSM: Diagnostic Statistical Manual of Mental Disorders; FSFI: Female Sexual Function Index; SD: Standard deviation; SHBG: Sex hormone blinding globulin}

\section{Acknowledgements}

Not Applicable.

\section{Funding}

No funding was obtained for this study.

\section{Availability of data and materials}

The rataset used and analyzed during the current study is available from the corresponding author on reasonable request.

\section{Authors' contributions}

ML: Performing hormonal analytical tests and analyzing the results; help to carry out the manuscript, related readings, realization of the discussion. ES: Help with the statistical plan, coordinator of the study, related readings. MR: Design of the statistical plan; analysis of the data derived from the satisfaction questionnaires carried out by the patients; contribution to the design of the study protocol; help to carry out the manuscript, related readings, realization of the discussion. FL: Contribution to the design of the study protocol; help to carry out the manuscript, related readings, realization of the discussion. SP: General Project Management and Principal Investigator; final design of the study protocol; final revision of the statistical conclusions. He is the main author in the writing of the manuscript. DK: Giving rationale and writing answers to reviewer questions.

Reviewing the pre-final manuscript version and implementing the modifications coming from reviewer questions and suggestions. All authors read and approved the final manuscript.

\section{Ethics approval and consent to participate}

In Spain, only the clinical trials with medicines are regulated by Royal Decree 1090/2015, of December 4, and Regulation (EU) No. 536/2014, without there being regulations that regulate the trials of intervention in humans with food.

The Spanish Agency for Food Safety (AECOSAN), under the Ministry of Health and Consumption, is the Spanish entity that regulates food supplements and does not establish administrative procedures prior to conducting an intervention trial in humans with food.

Therefore, no approval of the ethics committee is needed since Libicare ${ }^{\oplus}$ is a food supplement.

However, the pilot study that has been presented has been carried out following a well-defined protocol, and in accordance with the main premises of Good Clinical Practices and the Declaration of Helsinki.

In this way, all the participants have been informed, before their inclusion, of the possible risks and benefits, and of all the aspects of the study and have signed an Informed Consent expressing their willingness to participate. Likewise, during the study, all adverse events were registered and followed, fulfilling the maximum premise of safeguarding the safety of patients.

\section{Consent for publication}

Not applicable.

\section{Competing interests}

Dr. Santiago Palacios. Honoraria or consultation fees and participation in a company sponsored speakeaker's bureau: Bayer Healthcare, Bioiberica, Ferrer, Exeltis, Gedeon Richter, Gynea, MSD, Novo Nordisk, Pfizer, Procare Health Iberia S.L., Serelys, Servier, Shionogi, Teva. He also is a member of the editorial board of BMC Women's Health journal. 
Dr. Fernando Losa. Consultant in Spain Bayer, Procare Health Iberia S.L., ECare You.

Mrs. Eva M. Soler, Dr. Marieta Ramirez and Dr. Mariella Lilue declare no competing interests. Danial Khorsandi Ph.D. Principal researcher of the Procare Health Iberia S.L. and he has financial ties with Procare Health Iberia S.L.

\section{Publisher's Note}

Springer Nature remains neutral with regard to jurisdictional claims in published maps and institutional affiliations.

\section{Author details}

'Gynaecology and Obstetrics Department, Palacios' Institute of Women's Health, C/Antonio Acuña 9, E-28009 Madrid, Spain. 'Procare Health Iberia, Medical department, Barcelona, Spain. ${ }^{3}$ University of Barcelona, Barcelona, Spain. ${ }^{4}$ Clínica de la Sagrada Familia, Barcelona, Spain.

\section{Received: 18 June 2018 Accepted: 11 April 2019}

\section{Published online: 30 April 2019}

\section{References}

1. Basson R, Berman J, Burnett A, DeRogatis L, Ferguson D, Fourcroy J, et al. Report of the international consensus development conference on female sexual dysfunction: definitions and classifications. J Urol. 2000;163:888-93.

2. Dennerstein L, Koochaki P, Barton I, Graziottin A. Hypoactive sexual desire disorder in menopausal women: a survey of Western European women. J Sex Med. 2006;3:212-22.

3. Leiblum SR, Koochaki PE, Rodenberg CA, Barton IP, Rosen RC. Hypoactive sexual desire disorder in postmenopausal women: US results from the Women's international Study of health and sexuality (WISHeS). Menopause. 2006;13:46-56

4. Moreira ED Jr, Glasser DB, Gingell C. Sexual activity, sexual dysfunction and associated help-seeking behaviours in middle-aged and older adults in Spain: a population survey. World J Urol. 2005;23:422-9.

5. Shifren JL, Monz BU, Russo PA, Segreti A, Johannes CB. Sexual problems and distress in United States women: prevalence and correlates. Obstet Gynecol. 2008;112:970-8.

6. Laumann EO, Paik A, Rosen RC. Sexual dysfunction in the United States: prevalence and predictors. JAMA. 1999;281:537-44

7. American Psychiatric Association. Diagnostic and statistical manual of mental disorders, $5^{\text {th }}$ edition, text revision. Washington, DC: American Psychiatric Press; 2013.

8. Davis SR, Guay AT, Shifren JL, Mazer NA. Endocrine aspects of female sexual dysfunction. J Sex Med. 2004;1:82-6.

9. Santoro N, Torrens J, Crawford S, Allsworth JE, Finkelstein JS, Gold EB, et al. Correlates of circulating androgens in mid-life women: the study of women's health across the nation. J Clin Endocrinol Metab. 2005;90:4836-45.

10. Turna B, Apaydin E, Semerci B, Altay B, Cikili N, Nazli O. Women with low libido: correlation of decreased androgen levels with female sexual function index. Int J Impot Res. 2005;17:148-53.

11. Schiffer $L$, Arlt W, Storbeck KH. Intracrine androgen biosynthesis, metabolism and action revisited. Mol Cell Endocrinol. 2018;465:4-26.

12. Steels E, Steele ML, Harold M, Coulson S. Efficacy of a proprietary Trigonella foenum-graecum L. of-husked seed extract in reducing menopausal symptoms in otherwise healthy women: a double-blind, randomized, placebo-controlled Study. Phytother Res. 2017;31(9):1316-22.

13. Shamshad Begum S, Jayalakshmi HK, Vidyavathi HG, Gopakumar G, Abin I, Balu M, Geetha K, Suresha SV, Vasundhara M, Krishnakumar IM. A novel extract of fenugreek husk (FenuSMART ${ }^{\mathrm{M}}$ ) alleviates postmenopausal symptoms and helps to establish the hormonal balance: a randomized, double-blind, placebo-controlled Study. Phytother Res. 2016;30(11):1775-84.

14. Rao A, Steels E, Beccaria G, Inder WJ, Vitetta L. Influence of a specialized Trigonella foenum-graecum seed extract (Libifem), on testosterone, estradiol and sexual function in healthy menstruating women, a randomised placebo controlled Study. Phytother Res. 2015;29(8):1123-30.

15. Ito TY, Polan ML, Whipple B, Trant AS. The enhancement of female sexual function with ArginMax, a nutritional supplement, among women differing in menopausal status. J Sex Marital Ther. 2006:32(5):369-78.

16. Polan ML, Hochberg RB, Trant AS, Wuh HC. Estrogen bioassay of ginseng extract and ArginMax, a nutritional supplement for the enhancement of female sexual function. J Women's Health (Larchmt). 2004;13(4):427-30.
17. Ito TY, Trant AS, Polan ML. A double-blind placebo-controlled study of ArginMax, a nutritional supplement for enhancement of female sexual function. J Sex Marital Ther. 2001;27(5):541-9.

18. de Souza KZ, Vale FB, Geber S. Efficacy of Tribulus terrestris for the treatment of hypoactive sexual desire disorder in postmenopausal women: a randomized, double-blinded, placebo-controlled trial. Menopause. 2016;23(11):1252-6.

19. Wiegel M, Meston C, Rosen R. The female sexual function index (FSFI): crossvalidation and development of clinical cutoff scores. J Sex Marital Ther. 2005;31:1-20

20. Rosen R, Brown C, Heiman J, et al. The female sexual function index (FSFI): a multidimensional self-report instrument for the assessment of female sexual function. J Sex Marital Ther. 2000:26:191-208.

21. Stephenson KR, Toorabally N, Lyons L, M Meston C. Further validation of the female sexual function index: specificity and associations with clinical interview data. J Sex Marital Ther. 2016 Jul 3;42(5):448-61.

22. Meston CM. Validation of the female sexual function index (FSFI) in women with female orgasmic disorder and in women with hypoactive sexual desire disorder. J Sex Marital Ther. 2003 Jan-Feb;29(1):39-46.

23. Revicki DA, Margolis MK, Bush EN, De Rogatis LR, Hanes V. Content validity of the female sexual function index (FSFI) in pre- and postmenopausal women with hypoactive sexual desire disorder. J Sex Med. 2011 Aug;8(8):2237-45.

24. Dennerstein L, Randolph J, Taffe J, Dudley E, Burger H. Hormones, mood, sexuality, and the menopausal transition. Fertil Steril. 2002;77(Suppl)4):S42-8.

25. Davis SR, Davison SL, Donath S, Bell RJ. Circulating androgen levels and selfreported sexual function in women. JAMA. 2005;294:91-6.

26. Goldstein I, Kim NN, Clayton AH, DeRogatis LR, Giraldi A, Parish SJ, Pfaus J, Simon JA, Kingsberg SA, Meston C, Stahl SM, Wallen K, Worsley R. Hypoactive Sexual Desire Disorder: International Society for the Study of Women's Sexual Health (ISSWSH) Expert Consensus Panel Review. Mayo Clin Proc. 2017:92(1):114-28.

27. Perelman MA. Female sexual dysfunction and the central nervous system. J Sex Med. 2007:4:257-9.

28. Hull EM, Lorrain DS, Du J, Matuszewich L, Lumley LA, Putnam SK, et al. Hormone-neurotransmitter interactions in the control of sexual behavior. Behav Brain Res. 1999;105:105-16.

29. Davis SR, Braunstein GD. Efficacy and safety of testosterone in the management of hypoactive sexual desire disorder in postmenopausal women. J Sex Med. 2012:9(4):1134-48.

30. Davis SR, Moreau M, Kroll R, Bouchard C, Panay N, Gass M, Braunstein GD, Hirschberg AL, Rodenberg C, Pack S, Koch H, Moufarege A, Studd J, APHRODITE Study Team. Testosterone for low libido in postmenopausal women not taking estrogen. N Engl J Med. 2008:359(19):2005-17.

31. Palacios S. Androgens and female sexual function. Maturitas. 2007:57(1):61-5.

32. Armeni E, Lambrinoudaki I. Androgens and cardiovascular disease in women and men. Maturitas. 2017:104:54-72.

33. Spark RF. Intrinsa fails to impress FDA advisory panel. Int J Impot Res. 2005;17:283-4.

34. Thorp J, Simon J, Dattani D, Taylor L, Kimura T, Garcia M Jr, Lesko L, Pyke R, DAISY trial investigators. Treatment of hypoactive sexual desire disorder in premenopausal women: efficacy of flibanserin in the DAISY study. J Sex Med. 2012;9(3):793-804

35. Simon JA, Kingsberg SA, Shumel B, Hanes V, Garcia M Jr, Sand M. Efficacy and safety of flibanserin in postmenopausal women with hypoactive sexual desire disorder: results of the SNOWDROP trial. Menopause. 2014:21(6):633-40.

36. English C, Muhleisen A, Rey JA. Flibanserin (Addyi): the first FDA-approved treatment for female sexual interest/arousal disorder in premenopausal women. P T. 2017:42(4):237-41

37. Aftab A, Chen C, McBride J. Flibanserin and its discontents. Arch Womens Ment Health. 2017;20(2):243-7.

38. Akhtari E, Raisi F, Keshavarz M, Hosseini H, Sohrabvand F, Bioos S, Kamalinejad M. Ghobadi Atribulus terrestris for treatment of sexual dysfunction in women: randomized double-blind placebo - controlled study. Daru. 2014;22:40.

39. Zhao J, Dasmahapatra AK, Khan SI, Khan IA. Anti-aromatase activity of the constituents from damiana (Turnera diffusa). J Ethnopharmacol. 2008;120(3): 387-93.

40. Wu AND, Li S, Cui W, Zu X, Du J, Wang F. Ginkgo biloba extract improves coronary blood flow in healthy elderly adults: role of endotheliumdependent vasodilation. Phytomedicine. 2008;15(3):164-9. 